\title{
Und ewig lockt die Arztserie
}

Erhard Taverna

Erhard Taverna

\begin{abstract}
Das medizinische Ensemble der Fernsehserien formiert eine Untergruppe der Seifenopern. Deren Fans identifizieren sich mit den Bildschirmfiguren, leiden und freuen sich mit ihnen und kommentieren die fiktionalen Handlungen, als ob sie real wären. Wenn eine Langzeitserie abgesetzt wird, reagieren die Zuschauer mit Entzugssymptomen, mit wütenden Leserbriefen, tiefer Enttäuschung und frustrierter Hilflosigkeit. Genau davon träumen alle Macher, die nach hohen Einschaltquoten über möglichst viele Jahre lechzen.
\end{abstract}

eine Serie, an deren Drehbücher der kürzlich verstorbene Autor von «Jurassic Parc» Michael Crichton mit geschrieben hat. «Grey's Anatomy» und «Dr. House» begeistern Millionen, harte Konkurrenz für eine Produktion wie «Tag und Nacht» des Deutschschweizer Fernsehens. Seit die in Glattfelden nachgebaute «Permanence» des Zürcher Hauptbahnhofs Anfang September auf Sendung ging, schrumpfen die Zuschauerzahlen, trotz guten bis sehr guten Urteilen vonseiten der Kritiker. Viel Herzblut fliesse da, meinte der Tages-

\section{«Tag und Nacht»: «Feinstes TV-Serienhandwerk» made in Switzerland kämpft um Zuschauerzahlen}

Eine «Schoggi-Soap» wie «Lüthi und Blanc» ist mit 288 Folgen der heimliche Massstab der schweizerischen Genrefamilie, während unter den Arztserien die Schwarzwaldklinik am meisten Zuschauer erreichte und bei der «Sachsenklinik» nach 10 Jahren und über 400 Folgen immer noch kein Ende in Sicht ist. Im internationalen Markt dominiert «Emergency Room»,

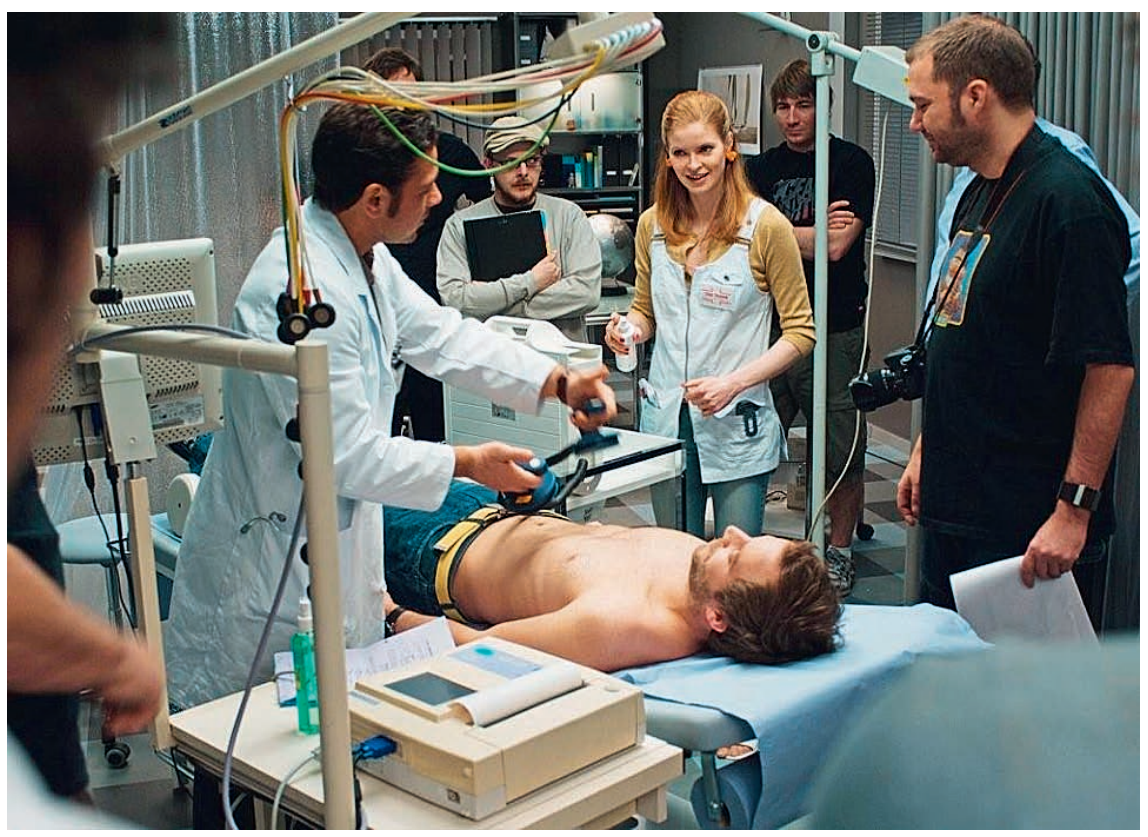

Wie war das noch mal? Leonardo Nigro alias Kardiologe Dr. med. Aebi und seine Praxisassistentin Connie Thalmann (gespielt von Lisa Ivana Brühlmann) auf dem Set beim Defibrillieren.
Anzeiger und feierte die «Versöhnung der Medizin und der hohen Krankenkassenprämien mit der Menschlichkeit». Heute ist immer noch vom «feinsten TV-Serienhandwerk» die Rede, spekuliert wird aber über falsche Sendezeiten und falsches Publikum, weil zu jung oder zu alt, sicher aber zu wenig zahlreich. Eine Aufzählung unglaubwürdiger oder übertriebener Szenen, die als «medizinische Lachnummern» abqualifiziert wurden, kritisierte angeblich unsachgemässe medizinische Handlungen. Natürlich muss übertrieben viel im Sprechzimmer passieren, damit diese kleine Kunstwelt in Schwung bleibt. Doch wie viel Realität braucht eine erfundene Handlung? Dieser schwierigen Aufgabe widmen sich echte Kollegen, die bei den Dreharbeiten für ein möglichst realitätskonformes Handeln der Schauspieler sorgen.

Einer aus diesem Beraterteam aus Ärzten und Krankenschwestern war der Allgemeinpraktiker und Vizepräsident der Zürcher Ärztegesellschaft Peter Tschudi aus Urdorf. Soeben sei die erste Staffel von 36 Folgen abgeschlossen, über eine Fortsetzung noch nichts entschieden. Seine Arbeit begann mit der Durchsicht der Drehbücher und Vorbesprechungen mit der Regie. Erst dann erfolgte die Instruktion der Schauspieler in den täglichen Untersuchungstechniken wie Auskultieren oder Palpieren, im Umgang mit EKG und Ultraschall oder in korrekter Aussprache von Fachausdrücken. Peter Tschudi ist beeindruckt von der vielseitigen Zusammenarbeit am Drehort und 


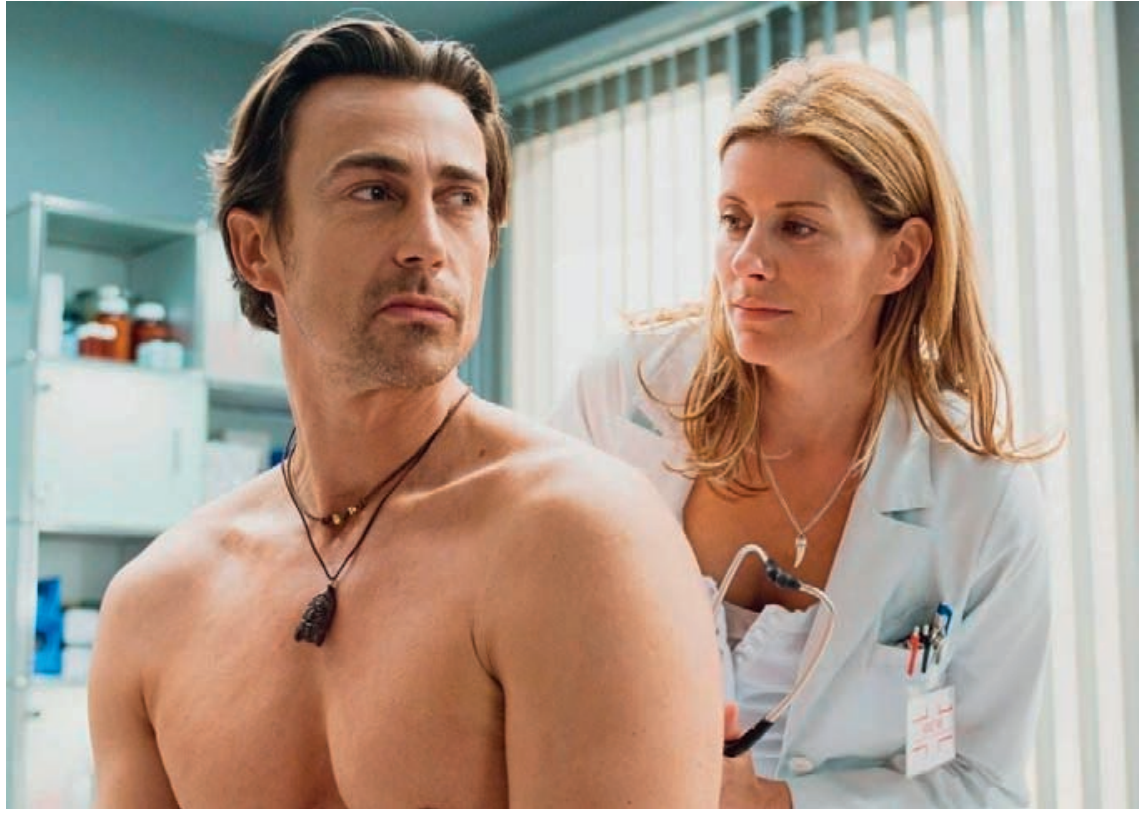

Auch attraktive Malariapatienten finden den Weg in die Permanence: Dr. med. Meret Frei (Sabina Schneebeli) untersucht Bruce Polson (Daniel Bernhardt).

dem ungeheuren Aufwand für wenige Filmminuten täglich. Das jeweilige Team habe stets unter enormem Zeitdruck, nach den minutiösen Vorgaben der Tagesdisposition, intensiv und seriös gearbeitet. «Die Medienschelte wegen kleinen medizinischen Fehlern ist ungerecht und kleinlich», findet er, denn schliesslich sei es wie in einem Theaterstück, wo auch die Toten nur gespielt und verschiedenste Handlungsstränge gleichgewichtig zu verknüpfen seien. Als regelmässiger Theater- und Kinogänger war die ungewohnte Arbeit im Studio für Peter Tschudi eine bereichernde Erfahrung, so dass er sich eine weitere Teilnahme vorstellen könne.
Inzwischen geben sich Sabina Schneebeli alias Dr. med. Frei und ihr Team alle Mühe, die Gunst des Publikums zu gewinnen. Dass die Serie kränkelt, liegt mehr am Drehbuch als an deren Fähigkeiten. Fehlende medizinische Detailtreue wird es nicht sein, wer dies wünscht, hat genügend Auswahl an Liveberichten aus dem Spital. Mag sein, dass die Akteure zu sehr mit sich selbst statt mit ihren Patienten beschäftigt sind. Doch das darf und muss wahrscheinlich so sein, grotesk, mörderisch, zynisch, obszön, gekünstelt und alles Mögliche sonst, nur nicht langweilig. Peter Tschudi ist ein bekennender Fan der Arztserie mit Dr. House. Warum dessen Diagnosequiz Fachleute wie Publikum gleichermassen fasziniere, könne er sich auch nicht erklären. Vielleicht liege es gerade daran, dass dieser Kollege überhaupt nicht dem Stereotyp des ethisch zertifizierten Vorbildes gleiche. Es kann auch daran liegen, dass grössere Budgets mehr Phantasie stimulieren. Am Freitagabend ist die Fernsehkonkurrenz auf allen Kanälen am grössten, ob es da genügt, mit einem gemächlicheren Bildrhythmus die Quoten zu ändern, bleibt abzuwarten. Im Rückblick wird jeder und jede genau wissen, was zum Erfolg oder Misserfolg beitrug. $\mathrm{Zu}$ bieder und kleinkariert werden die einen sagen, $\mathrm{zu}$ frech und $\mathrm{zu}$ wenig realistisch, werden andere meinen. Bleibt die Frage, was an diesen vielen Serien so verlockend bleibt. Ein Schulhaus als Drehort gäbe mindestens soviel her.

PS: Operation gelungen, Patient gestorben. $\mathrm{Zu}$ einer zweiten Staffel wird es nicht mehr kommen, es bleibt bei den restlichen Folgen der nächsten Monate. 